\title{
1918: Zwischen Weltkrieg und Revolution. Ein Entscheidungsjahr für Deutschland
}

\author{
Eine Kabinettausstellung
}

Anlässlich des 100. Jahrestags zum Ende des Ersten Weltkriegs und zur Novemberrevolution zeigte die WLB vom 11. Oktober bis 29. November 2018 die Ausstellung „1918: Zwischen Weltkrieg und Revolution - Ein Entscheidungsjahr für Deutschland". Die Schau war Teil einer im Rahmen der Französischen Wochen mit dem Institut français Stuttgart organisierten Veranstaltungsreihe zum Kriegsende 1918. Im Institut war zeitgleich die Partnerausstellung "Neubeginn in Stuttgart und Straßburg" zu sehen.

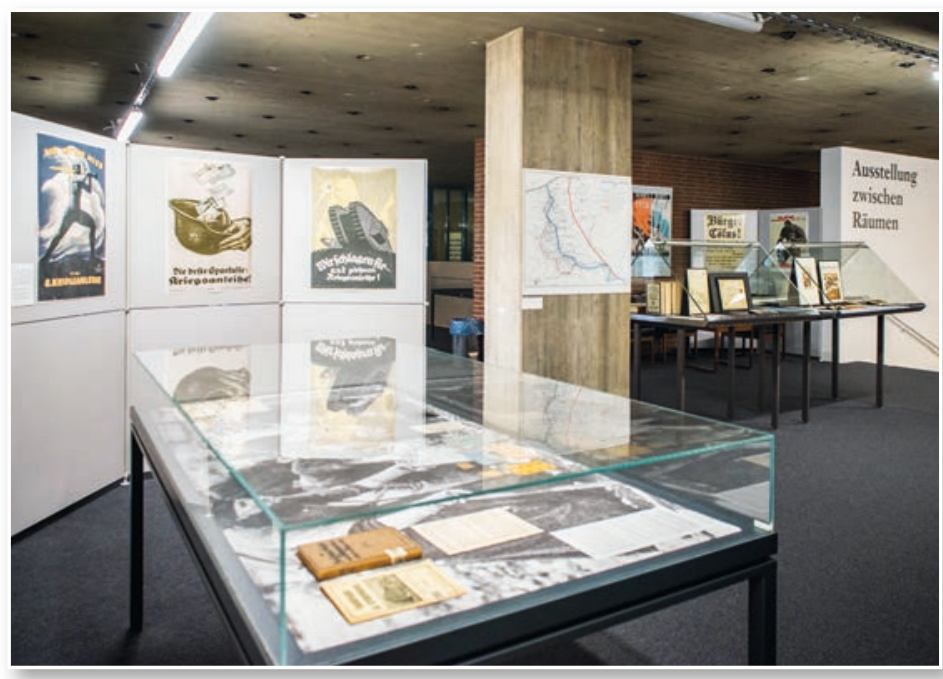

Abb. 1: Blick in die Ausstellung

Inspiriert von dem Buch „1918. Die Deutschen zwischen Weltkrieg und Revolution" " widmete sich die von Dr. Christian Westerhoff und Irina Renz konzipierte und mit Hilfe von Dr. Ida Danciu umgesetzte Kabinettausstellung den Ereignissen und Bedingungen des letzten Kriegsjahres. Ein Jahr, das für die Deutschen mit großen Hoffnungen begann, die im Laufe der Zeit aber in bittere Enttäuschung umschlugen, und das mit Ungewissheit über die weitere Entwicklung endete. Der Friedensschluss mit Russland, die große Offensive im Westen, für die noch einmal alle Kräfte gebündelt wurden, ein Vorrücken auf Paris nach vier
Jahren Stellungskrieg, das Ende des Vormarsches und die Gegenoffensive der Alliierten, der Hunger und die zunehmend schlechtere Stimmung an der Heimatfront, die schließlich in der Revolution mündete, das Ende der Monarchie und des Krieges, ein Waffenstillstand, der einer Kapitulation glich, die Heimkehr der Soldaten, der demokratische Neuanfang Deutschlands, die Entstehung einer neuen Weltordnung: All diese Entwicklungen vollzogen sich wie in einem Brennglas innerhalb von nur zwölf Monaten. Die enge Abfolge der Ereignisse war für viele Zeitgenossen eine emotionale Achterbahnfahrt. In kurzer Zeit kam es zu großen Zäsuren mit sehr weitreichenden Folgen. Mit dem Ende des Ersten Weltkriegs und der Novemberrevolution begann der Kampf um die Grenzen und die Demokratisierung Deutschlands - Auseinandersetzungen, die das 20. Jahrhundert bestimmen sollten.

Anhand von teilweise erstmals gezeigten Stücken aus den umfangreichen Sammlungen der Bibliothek für Zeitgeschichte (BfZ) gab die Ausstellung in sieben Themenblöcken Einblick in die Voraussetzungen, Krisen, Wendepunkte und Folgen. Zu sehen waren Bücher, Broschüren und Zeitungen, Plakate, Fotografien, Postkarten und Flugblätter, aber auch untypisches Bibliotheksgut wie Lebensmittelmarken, Granatsplitter und Orden. Ergänzt wurde die Auswahl durch außergewöhnliche Leihgaben.

Den Anfang der Ausstellung bildete die militärische und politische Ausgangslage: An der Ostfront schwiegen infolge der Russischen Revolution seit Dezember 1917 die Waffen. Viele Deutsche schöpften neuen Mut und hofften, dass es doch noch zu einem siegreichen Ende des Krieges kommen könnte. Im selben Jahr waren jedoch auch die USA auf Seiten der Alliierten in den Krieg eingetreten. Dadurch gerieten das Deutsche Reich und seine Verbündeten im Laufe des Jahres 1918 
immer mehr in die Defensive. Bei der Illustrierung dieser Vorgeschichte bot es sich an, die vielfältigen Bestände an Propagandamaterialien zu nutzen sowie ein Fotoalbum mit Aufnahmen von den Friedensverhandlungen in Brest-Litowsk aufzuschlagen.

Die Lage an der Westfront, wo nach dem Willen der Obersten Heeresleitung im Frühjahr 1918 die Entscheidungsschlacht stattfinden sollte, veranschaulichten im nächsten Abschnitt zahlreiche Fotos. Ein Hintergrundbild mit Ruinen und Bombentrichtern zeigte, welche Spuren der Krieg nach vier Jahren in der Frontlandschaft hinterlassen hatte. In dieser Mondlandschaft sollte der "Letzte Hieb", der auf den ausgehängten Kriegsanleihe-Plakaten beworben wurde, gegen die Alliierten geführt werden. Einen Bezug zur Gegenwart stellte eine Leihgabe der Stadt Backnang her; ein Kasten mit den Überresten der Ausrüstung eines Soldaten: rostige Munition, zerfallene Uniformteile, eine zerbröckelte Gasmaske. Der Backnanger Wilhelm Härer hatte im Rahmen der alliierten Gegenoffensive im Oktober 1918 den Kontakt zur Truppe verloren und galt seitdem als vermisst. Erst 2009 konnte anhand der im Argonner Wald ebenfalls ausgegrabenen Erkennungsmarke Härers Schicksal endlich geklärt werden.

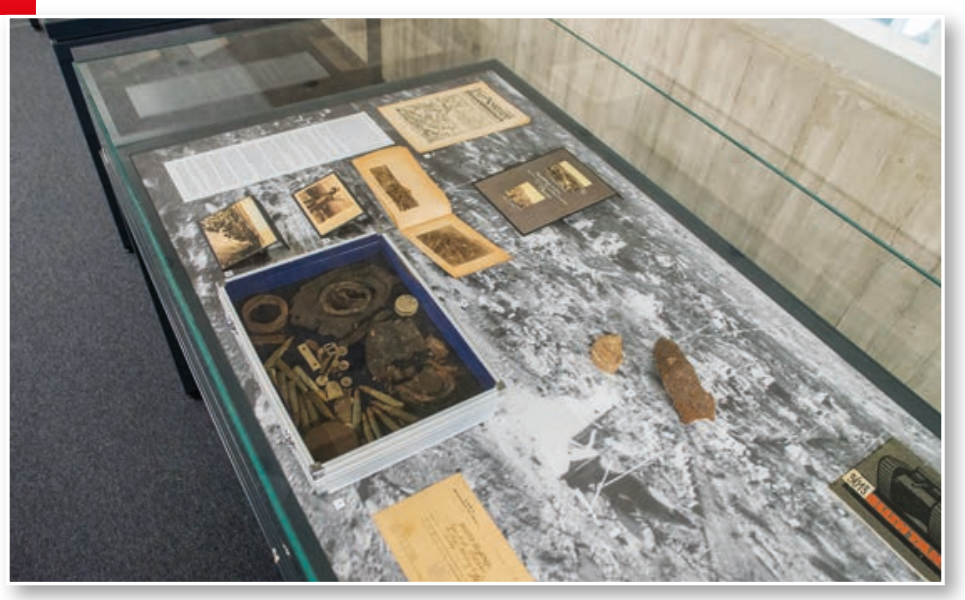

Abb. 2: Vitrine zur Lage an der Front

Im letzten Kriegsjahr verschärfte sich die Lage auch für die Zivilbevölkerung in der Heimat. Aufgrund einer britischen Seeblockade erlebten die Deutschen seit 1914 eine harte Zeit des Mangels, der immer größer wurde, je länger der Krieg dauerte. Bei Kriegsende litten große Teile der Bevölkerung an Unterernährung, ca. 800.000 Menschen starben während des Kriegs in Deutschland an
Hunger. Die Lebensmittelknappheit, die in der Ausstellung unter anderem durch Rationierungsmarken aus Stuttgart dokumentiert wurde, entwickelte sich für breite Kreise zum beherrschenden Thema. Brennnesseln, Stroh, Torf und Eicheln, die das Industriemuseum Lohne zur Verfügung gestellt hatte, vermittelten anschaulich, welch unterschiedliche Materialien während des Ersten Weltkriegs als Ersatzstoffe für fehlende Güter des alltäglichen Bedarfs herhalten mussten.

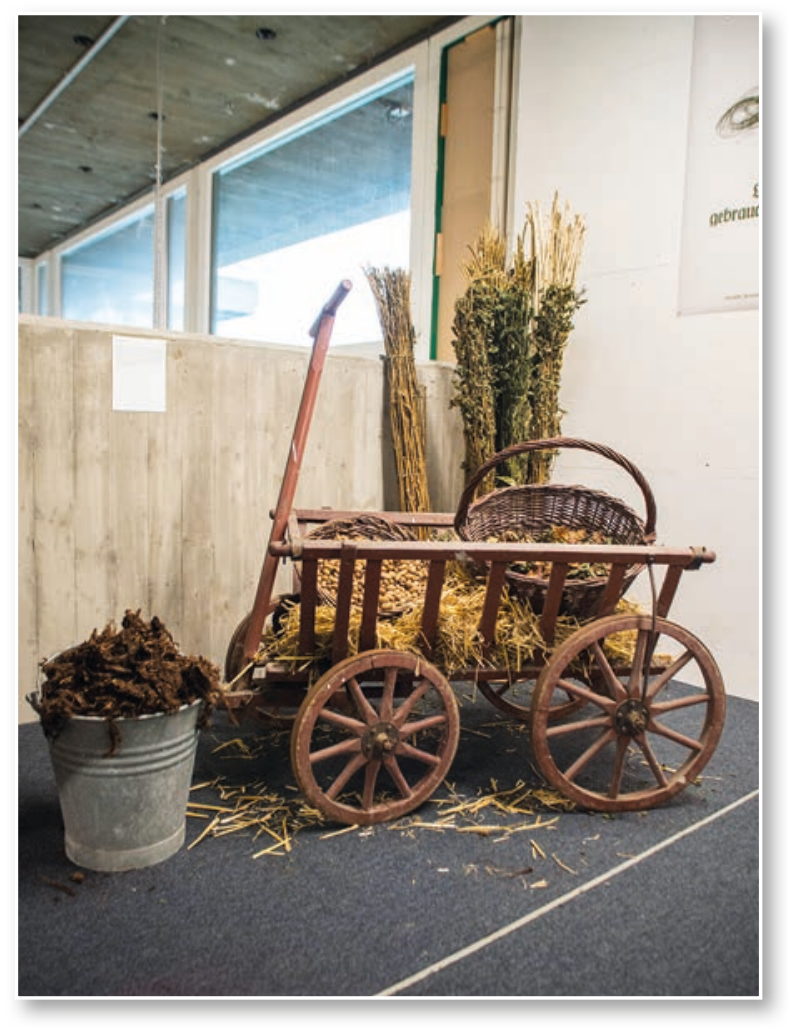

Abb. 3: Brennnesseln, Torf, Stroh und Eicheln. Ersatzstoffe für fehlende Güter des täglichen Bedarfs

Die Stimmung der Bevölkerung, die sich im letzten Kriegsjahr zwischen Durchhaltewillen und Friedenssehnsucht bewegte, thematisierte der folgende Ausstellungsteil. Orden, Fotos und Postkarten zeugten davon, wie die militärische und politische Führung bis zuletzt versuchte, die Bevölkerung davon zu überzeugen, dass nur ein deutscher Sieg zu einem annehmbaren Frieden führen werde. Insbesondere die Arbeiterschaft war jedoch zunehmend kriegsmüde.

Ausgehend von Matrosenaufständen in Kiel und Wilhelmshaven verbreitete sich Anfang November 1918 die Revolution über ganz Deutschland. Die Bevölkerung forderte in zahlreichen Städten ein Ende des Krieges, den Sturz der Monarchie und die Demokratisierung Deutschlands. Alle deutschen Könige und Fürsten beugten sich 
dem Druck der Straße und dankten ab. Auch der württembergische König Wilhelm II. verzichtete auf den Thron, nachdem in Stuttgart Revolutionäre das Wilhelmspalais, das heutige Stadtpalais, bedroht hatten. Zum Thema Revolution konnte die WLB auch auf spannende Leihgaben aus Backnang zurückgreifen. Dr. Roland Idler stellte Fotos und einen Passierschein seines Vaters Gottlieb zur Verfügung, der am Matrosenaufstand in Kiel teilgenommen hatte.

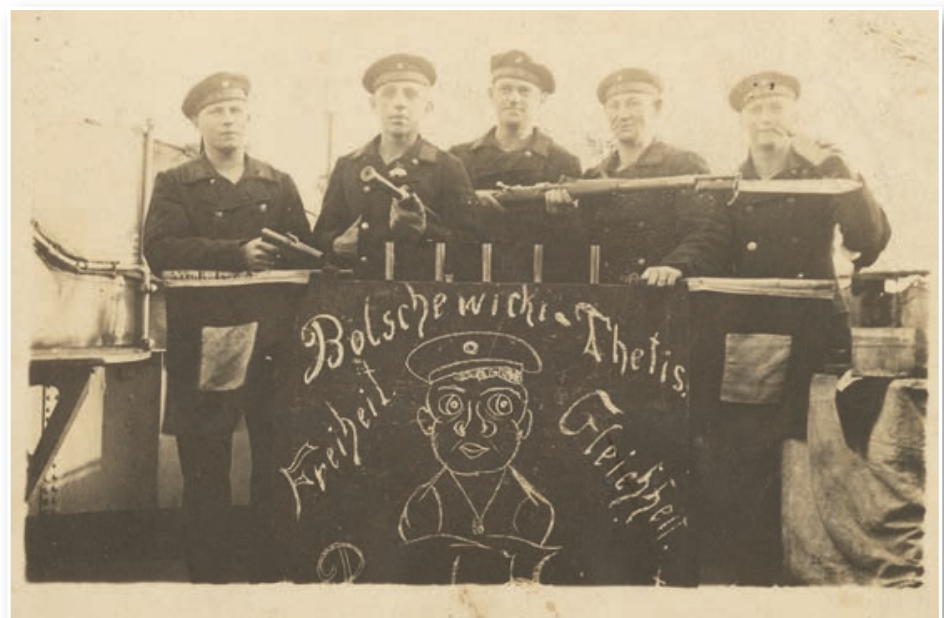

Abb. 4: Gottlieb Idler (Mitte) als "Bolschewik" des Kreuzers Thetis während des Matrosenaufstands

Am 11. November 1918 unterzeichnete der Württemberger Matthias Erzberger für das Deutsche Reich in Compiègne den Waffenstillstand, nachdem die Oberste Heeresleitung Ende September eine Fortführung des Kampfes für sinnlos erklärt hatte. Das plötzliche Eingeständnis der Niederlage und die harten Bedingungen des Waffenstillstandsabkommens stellten für viele Deutsche einen Schock dar. Die heimkehrenden Soldaten wurden in vielen Städten als „im Felde unbesiegt" begrüßt. Ein damals unter den Soldaten verbreitetes Flugblatt zeigte Verlautbarungen des Chefs der Obersten Heeresleitung Paul von Hindenburg und des Reichskanzlers Friedrich Ebert.

Ein Ausblick auf die weitreichenden Folgen dieses „Entscheidungsjahres" bildete den Abschluss der Ausstellung. Die Verbitterung über das von vielen Deutschen unerwartete und nicht verstandene Ende des Krieges führte zur Suche nach Schuldigen, die sich in einer Flut von Publikationen niederschlug. Die "Dolchstoßlegende", welche nicht das Militär, sondern die Revolutionäre vom Herbst 1918 für die Niederlage verantwortlich machte, stellte für die Weimarer Republik eine schwere Hypothek dar. Auch das Bemühen um eine Revision des Versailler Vertrages, dem sich auch die BfZ - die damalige Weltkriegsbücherei - verschrieb, einte die Deutschen nicht, sondern vertiefte die politischen Gräben noch zusätzlich, galten die demokratischen Politiker doch bald als Erfüllungsgehilfen der Alliierten.

Das letzte Exponat war ein großes gerahmtes Wandbild aus der Weimarer Republik, das Gottlieb Idler aus Backnang an seine Dienstzeit in der Marine während des Ersten Weltkriegs erinnerte. Mit Symbolen des Kaiserreiches dekoriert, verweist das Bild, auf dem die Soldatentreue beschworen wird, auf die schwierige Erinnerung an die revolutionären Ereignisse des Jahres 1918.

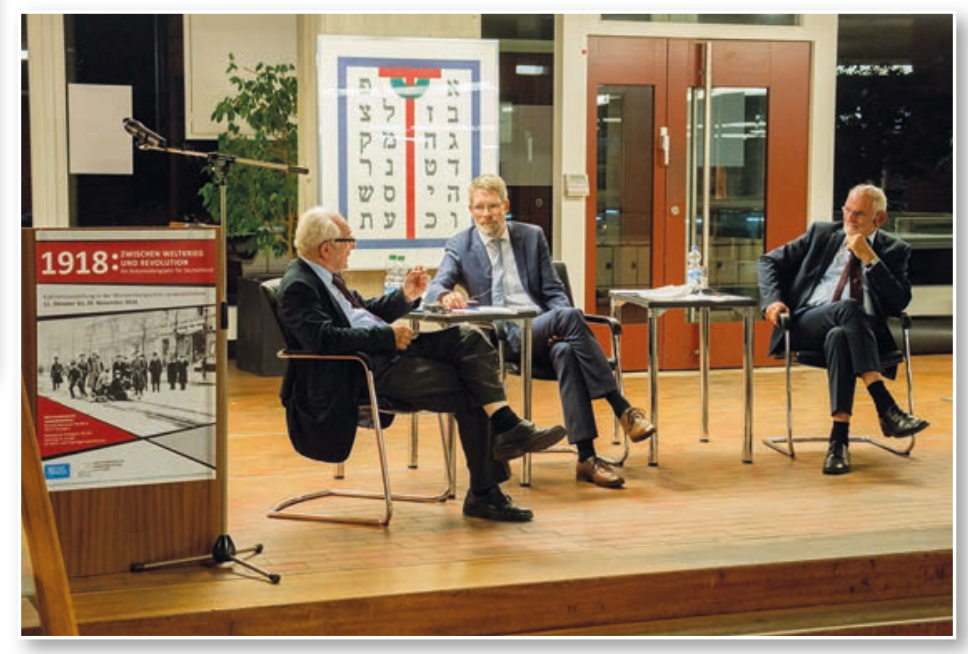

Abb. 5: Podiumsdiskussion im Rahmen der Ausstellungseröffnung: Georges-Henri Soutou, Christian Westerhoff und Gerd Krumeich (v.I.n.r.)

Die Ausstellung wurde am 11. Oktober 2018 mit einer Podiumsdiskussion eröffnet. Nach der Begrüßung durch die stellvertretende Direktorin, Martina Lüll, und einer Einführung durch den Leiter der BfZ, Dr. Christian Westerhoff, diskutierten Professor Dr. Gerd Krumeich (Freiburg) und Professor Dr. Georges-Henri Soutou (Paris) leidenschaftlich aus deutscher und französischer Sicht über "Hoffnungen, Illusionen und Realitäten 1918/1919“. Etwa 100 Besucher verfolgten die lebhafte Diskussion und sahen sich im Rahmen des anschließenden Empfangs die Ausstellung an. 


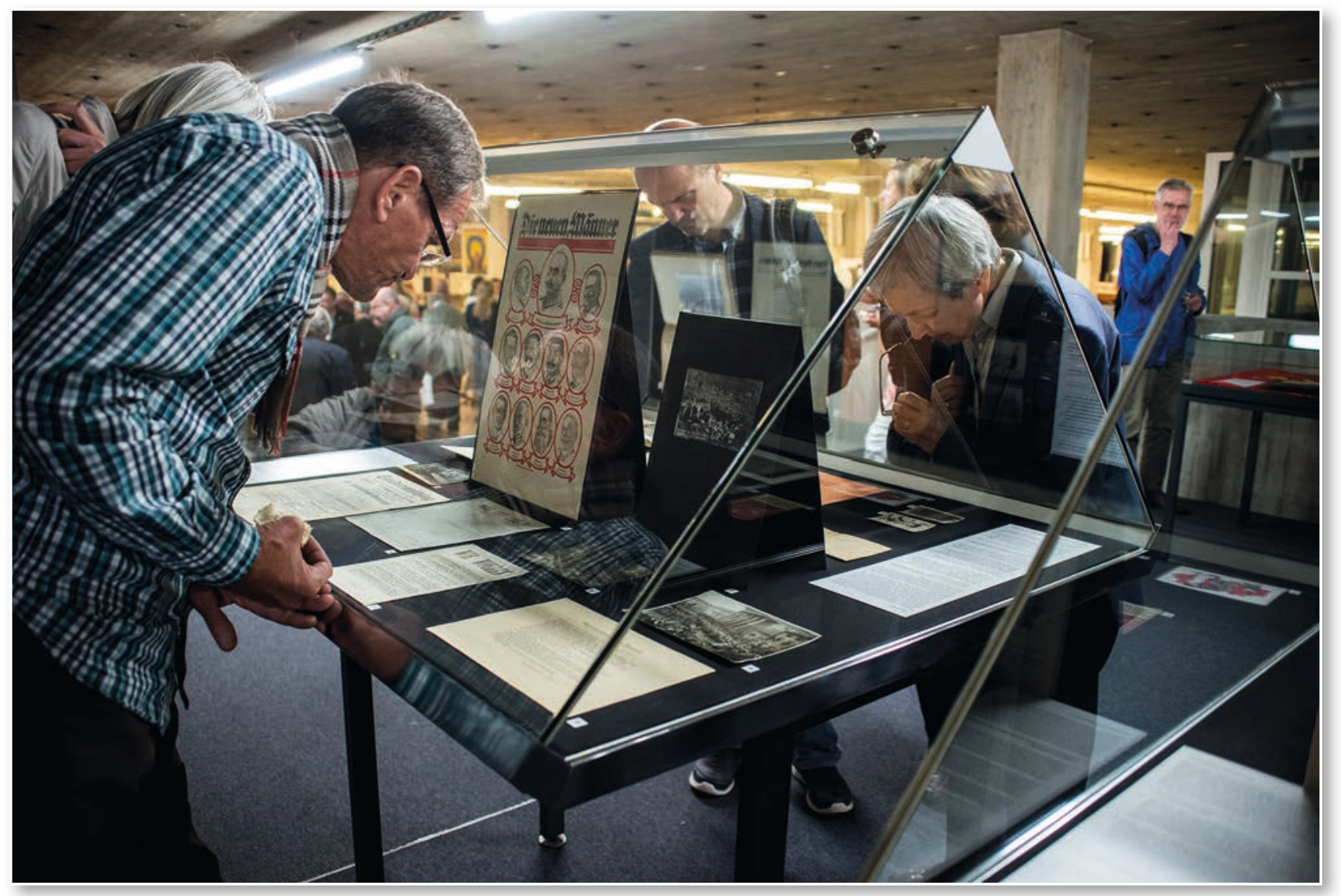

Abb. 6: Besucher in der Ausstellung

Über die Ausstellung wurde in verschiedenen Formaten in den Medien berichtet. Artikel in Zeitungen ${ }^{2}$ und Zeitschriften ${ }^{3}$ wiesen auf „1918. Zwischen Weltkrieg und Revolution" hin. Der SWR strahlte eine Fernsehsendung zum Ende des Ersten Weltkriegs aus, in der die Schau der WLB prominent vertreten war. ${ }^{4}$
Im Anschluss an die Präsentation in der WLB war die Ausstellung vom 4. bis 13. Dezember 2018 in der Stadtbibliothek Stuttgart zu sehen. An beiden Veranstaltungsorten nahmen zahlreiche Besuchergruppen an Führungen teil. Es ist angedacht, die Ausstellung darüber hinaus 2019 in Backnang zu zeigen.

Christian Westerhoff

2) Stuttgarter Zeitung vom 19.9.2018 und 5.12.2018; Stuttgarter Nachrichten vom 16.10.2018; Südwest-Presse vom 8.10.2018; Backnanger Kreiszeitung vom 6.10.2018, 10.11.2018 und 6.12.2018.

3) Focus online, Stuttgart Regional, 26.9.2018; Momente, Beiträge zur Landeskunde von Baden-Württemberg, 4/2018, S. 29.

4) SWR aktuell, 10.11.2018, https://www.swr.de/swraktuell/baden-wuerttemberg/Weltkriegsende, av-01069556-100.html. 